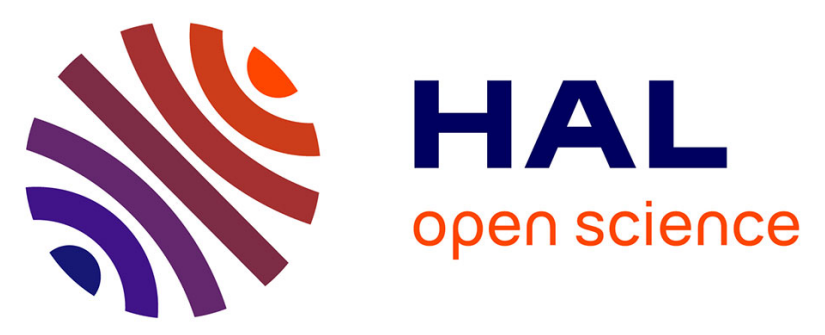

\title{
First-Body Versus Third-Body: Dialogue between an Experiment and a Combined Discrete and Finite Element Approach
}

\author{
Mathieu Renouf, Viet-Hung Nhu, Aurélien Saulot, Francesco Massi
}

\section{To cite this version:}

Mathieu Renouf, Viet-Hung Nhu, Aurélien Saulot, Francesco Massi. First-Body Versus Third-Body: Dialogue between an Experiment and a Combined Discrete and Finite Element Approach. Journal of Tribology, 2014, 136 (2), pp.021104. 10.1115/1.4026062 . hal-01006459

\section{HAL Id: hal-01006459 \\ https://hal.science/hal-01006459}

Submitted on 16 Jan 2017

HAL is a multi-disciplinary open access archive for the deposit and dissemination of scientific research documents, whether they are published or not. The documents may come from teaching and research institutions in France or abroad, or from public or private research centers.
L'archive ouverte pluridisciplinaire HAL, est destinée au dépôt et à la diffusion de documents scientifiques de niveau recherche, publiés ou non, émanant des établissements d'enseignement et de recherche français ou étrangers, des laboratoires publics ou privés. 


\section{First-Body Versus Third-Body: Dialogue Between an Experiment and a Combined Discrete and Finite Element Approach}

The present paper proposes to analyze relations between the behavior of two bodies in contact (local stress and vibration modes) and the rheology of third-body particles. Experiments are performed on a system composed of a polycarbonate disk in contact with a steel cylinder, where birefringent property of polycarbonate allows us to observe shear-stress isovalues. Multiscale numerical simulations involve the coupling between fi-nite elements and discrete elements to model simultaneously nonhomogeneous third-body flows within a confined contact and dynamical behavior of the bodies in contact. Compar-isons between experiments and simulations are performed on the dynamic response of the system, the stress distribution, as well as the evolution of third-body particles within the contact. Such comparisons exhibit not only qualitative results but also quantitative ones and suggest a new approach to study in deeper third-body rheology.

Keywords: DEM-FEM coupling, third-body flows, photoelasticimetry

Mathieu Renouf

Associate Researcher

LMGC,

University of Montpellier 2,

CNRS,

Montpellier F-34096, France

e-mail: Mathieu.Renouf@univ-mont2.fr

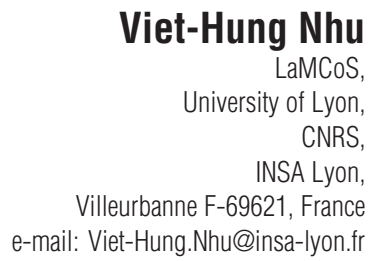

\section{Introduction}

When two bodies in contact (called first bodies [1]) are submitted to tribological conditions (contact pressure and shear velocity simultaneously), several phenomena occur at the scale of the contact interface: surface transformation [2], heat generation [3], stress localization [4], third-body flows [5], etc. Moreover, the relative motion related to tribological conditions can lead to instability states, such as modal dynamic instabilities, stick-slip, or stickslip-separation up to several kHz. Such phenomena, characterized by large system vibrations, was studied analytically [6,7] as well as numerically by using the finite element method (FEM) using, for example, a constant Coulomb friction coefficient [8] possibly coupled with regularization time, as in Prakash-Clifton law [9].

Nevertheless, such models do not account for the third-body flow inside the contact and its interactions with contact dynamics. On the other side, if previous third-body simulations have occulted first-body behavior [10,11], nowadays it is possible to couple FEM with discrete element method (DEM) to compensate limitation of past models [12-14].

Based on such an approach, the purpose of the present work is to exhibit quantitative numerical results accounting for both firstbody vibrations and third-body rheology. This system is able to reproduce modal dynamic instabilities, induced by frictional forces, giving origin to large harmonic system vibrations up to the appearance of local sticking and detachment zones along the contact interface. To exhibit quantitative results, an experimental setup has been developed to obtain different levels of comparisons (system vibrations, local stresses, and third-body rheology). After a presentation of the experimental setup exposed in Sec. 2, the headlines of the numerical approach used in this paper are presented in Sec. 3. Then, a discussion around experimental and numerical results is performed in Sec. 4 before a final section concluding the paper.

\section{Experimental Approach}

2.1 Setup. The experiment (cf. Fig. 1) has been developed in order to track simultaneously first-body vibrations and third-body rheology under frictional contact conditions. The apparatus is composed of a polycarbonate holed disk tied on its outer circumference and an expandable rotating cylinder made of stainless steel. The contact pressure, denoted $P$, between the two main elements is controlled by a static radial expansion of the steel cylinder. When the wanted pressure is reached, the cylinder is put in rotation by a motor with a constant angular velocity, denoted $\omega$.

As the disk is constituted of polycarbonate (a hard photoelastic material) with birefringence properties, it is possible to perform a photostress analysis of the isochromatics to obtain a dynamic visualization of the deviatoric stresses in the disk (i.e., $\left|\sigma_{1}-\sigma_{2}\right|$ ) during the friction process. This analysis depends on the Brewster constant, which shows correlation between the optical path difference between vibrations along two perpendicular axes (with minimal and maximal velocities) and stresses that gave rise to this difference. The isochromatics can be directly compared to the stresses computed in numerical simulations. To improve their legibility, a monochromatic filter with a wavelength, $\lambda$, equal to $546 \mathrm{~nm}$ has been used to select only the isochromatic proportional to $\tau_{\max }$ equal to $0.35 \mathrm{MPa}$ (the black lines in Fig. 3). A thermocouple has been added, close to the contact interface, to control temperature variations. During the experimental tests presented in Secs. 2.2, 2.3, and 2.4 the value of this temperature stays comparable to the ambient one. Consequently, there is no thermal effect 


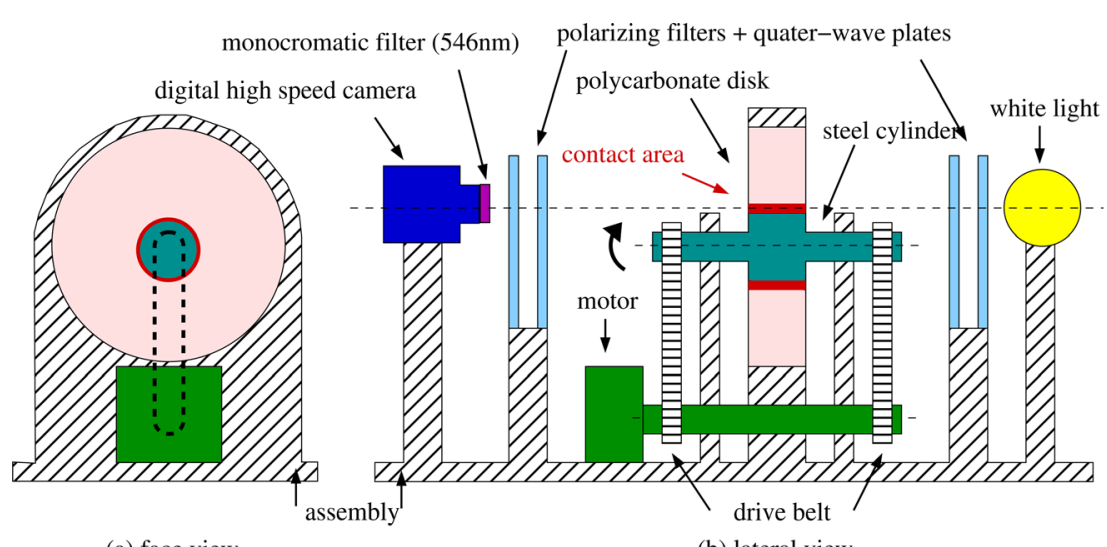

(a) face view

(b) lateral view

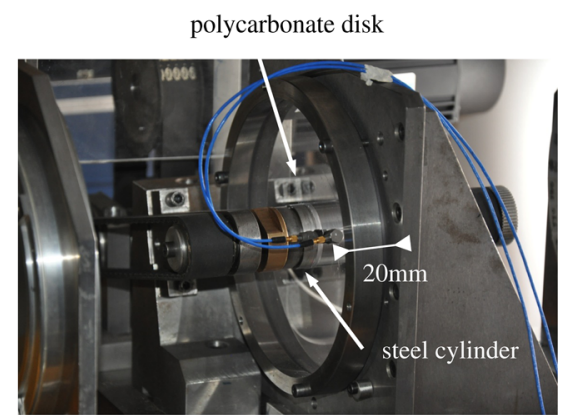

(c) photograph of

the experimental setup

Fig. 1 Sketch of the (a) face and (b) lateral views of the experimental setup and (c) photograph of the experimental setup

when isochromatic is observed. Both material and geometrical characteristics are summarized in Table 1.

To account for the high frequencies of the unstable vibrations of such system, a high-speed video camera has been used with a sampling rate compatible with the frequency of the instability (i.e., 20,000 fps at $640 \times 480$ pixels). In addition, both in-plane (radial) and out-of-plane accelerations of the disk are recorded with two piezoelectric accelerometers with a sensitivity of $10 \mathrm{mV} / \mathrm{g}$ and radially positioned at about $20 \mathrm{~mm}$ from the diskcylinder interface (cf. Fig. 1). The radial expansion of the steel cylinder is monitored by two eddy current transducers, diametrically opposite, with a sensitivity of $8 \mathrm{mV} / \mu \mathrm{m}$.

Finally, the rotational velocity of the cylinder is imposed and controlled by a brushless motor linked to an optical encoder. To maintain a constant velocity all along the experiment, the engine torque evolves automatically, thanks to a feedback loop linked to a velocity encoder. Such evolution is directly linked to the reaction torque of the contact interface. Data have been exploited qualitatively to see the tendency of such evolution. The constant of proportionality of the torque transmitted could not be measured or computed easily. The constant of proportionality of the velocity transmission between the motor and the axle has been computed with the number of teeth of the pulley and is equal to a reduction coefficient of 2.14 .

2.2 Identification of Instability States. Relative motion between solids in frictional contact can give origin of different stable or unstable scenarios (continuous sliding, stick-slip, mode coupling instability, etc.) as a function of the system and contact parameters [15]. In this paper, the experimental and numerical systems are set to give rise to mode-coupling instabilities due to the unstable interaction between two system modes that are coupled by the contact forces [15]. This kind of contact instability is characterized by large harmonic vibrations of the system, at one of the system natural frequencies, which increase exponentially up to a limit cycle due to the rise of contact nonlinearities [15].

Table 1 Material and geometrical characteristics of the main elements of the experimental setup

\begin{tabular}{lcc} 
& Disk & Cylinder \\
\hline Young's modulus $(\mathrm{GPa})$ & 2.3 & 205 \\
Poisson ratio & 0.38 & 0.3 \\
Density $\left(\mathrm{kg} / \mathrm{m}^{3}\right)$ & 1200 & 7800 \\
Internal radius $(\mathrm{mm})$ & 20.5 & - \\
External radius $(\mathrm{mm})$ & 92.5 & 20.5 \\
Thickness $(\mathrm{mm})$ & 10 & - \\
Brewster constant $\left(\mathrm{Pa}^{-1}\right)$ & $78 \times 10^{-12}$ & - \\
\hline
\end{tabular}

After a calibration of the experimental setup and a first parametrical analysis (radial expansion, rotational speed, and contact surface properties) [16,17], an identification of the instability states is performed, with or without creation of third-body, using spectrogram of in-plane and/or out-of-plane acceleration.

Figure 2 shows the spectrogram of a first experiment performed with a radial expansion of $30 \mu \mathrm{m}$ and a rotation speed $\omega=0.1 \mathrm{~m} / \mathrm{s}$. The $\mathrm{X}$-axis corresponds to the scale of frequency and the $\mathrm{Y}$-axis to the time evolution, while the color scale refers to the intensity of acceleration. With such a graph, for a given frequency, it is possible to see its evolution in time and, for a given time, which frequencies are involved. During the duration of the first experiment, the applied conditions do not still lead to the creation of third-body particles. The spectrogram (cf. Fig. 2(a)) is characterized by a main frequency at $7.5 \mathrm{kHz}$ with its super harmonics. The harmonic behavior of the vibrations at a natural frequency of the system is characteristic of autoexcited vibrations due to modal coupling [15]. Note that the value of the main frequency can have slight variations from one disc to another. Such behavior is due to the sensitivity to both the boundary conditions applied to the external side of the disc and the machining tolerance on the inner diameter of the disc lower than $5 \mu$ m diametrally. Then, the main frequency of the instability state reproduced experimentally evolves in the range of $7.5 \mathrm{kHz} \pm 1.5 \mathrm{kHz}$ but with a similar shape of the excited (unstable) mode.

After a period of running in with the same boundary conditions, the creation of third-body particles starts (i.e., an increase of thirdbody flow). A progressive competition takes place between the instability states, occurring since the beginning of the experiment, and the birth of third-body particles in the contact, occurring later during the experiment. If the number and the size of third-body particles are small enough, nothing happens on the spectrogram. If the third-body particles reach a critical size, a transition between an unstable state and a stable state occurs as highlighted by Fig. 2(b), where the unstable vibrational frequency at $7.5 \mathrm{kHz}$ disappears during the test.

2.3 Local Stresses. Due to the property of birefringence properties of the polycarbonate disk, a photostress analysis of the isochromatics is performed to obtain the evolution of the deviatoric stresses in the disk (i.e., $\left|\sigma_{1}-\sigma_{2}\right|$ ) during the rotation of dilated the steel cylinder (cf. Fig. 3).

Initially, the deviatoric stress $\left(\tau_{\max }\right)$ is close to $0.7 \mathrm{MPa}$ (two fringes). When the instability state occurs, due to the contact forces, oscillations of the stress distribution are observed. Consequently, $\tau_{\max }$ evolves in a range from $0.7 \mathrm{MPa}$ (two fringes) to 1.4 $\mathrm{MPa}$ (four fringes) (cf. Fig. 3(a)).

Then, the instability state disappears and a large amount of large third-body particles (up to $20 \mathrm{~mm}$ in length and $50 \mu \mathrm{m}$ thick) is observed in the contact (cf. Fig. 4). The presence of these 
(a)

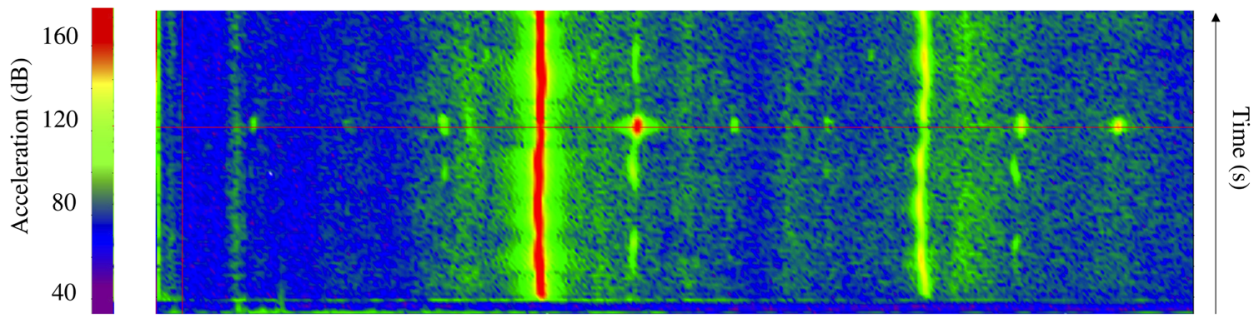

(b)

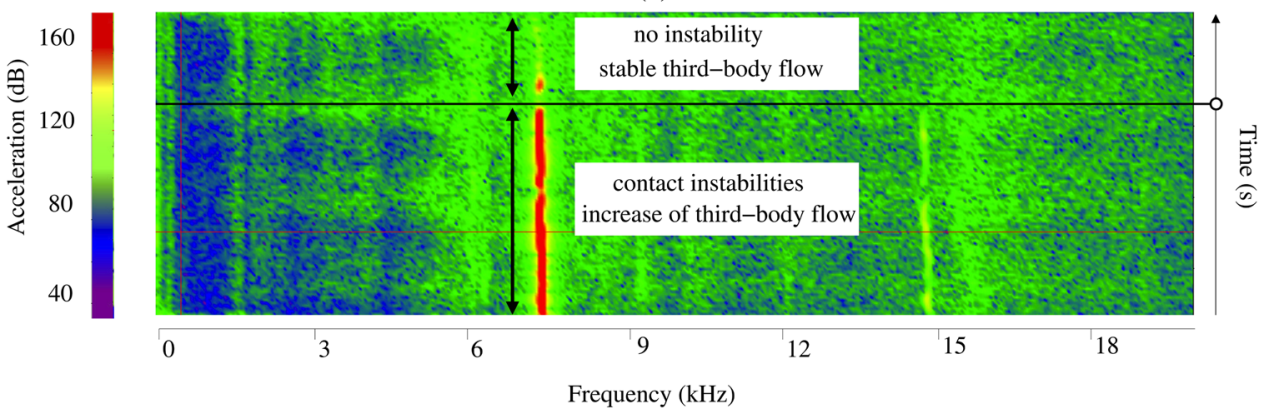

Fig. 2 Spectrogram of instability states (a) during the increase of third-body flow and $(b)$ with a stable third-body flow for a radial expansion of $20 \mu \mathrm{m}$ and a rotation speed $\omega=0.2 \mathrm{~m} / \mathrm{s}$

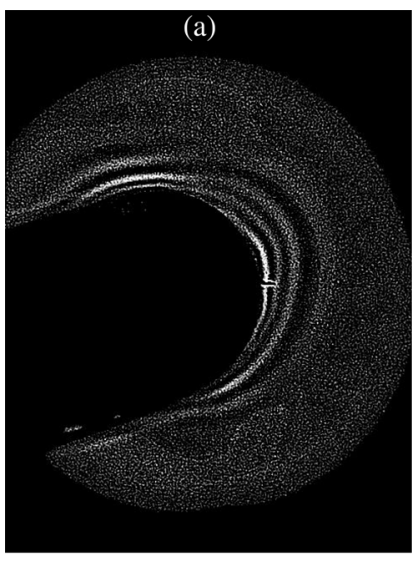

$\mathrm{t}=0 \mathrm{~s}$

instability states without third-body

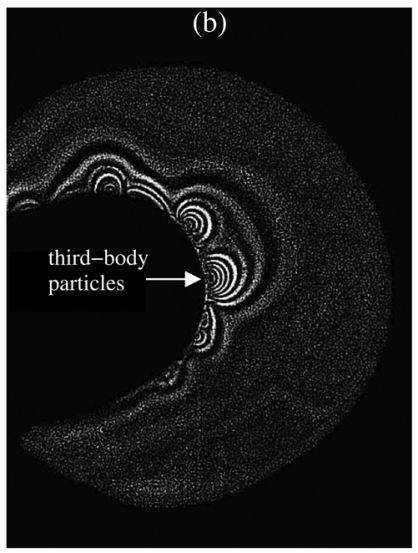

$\mathrm{t}=2 \mathrm{~s}$

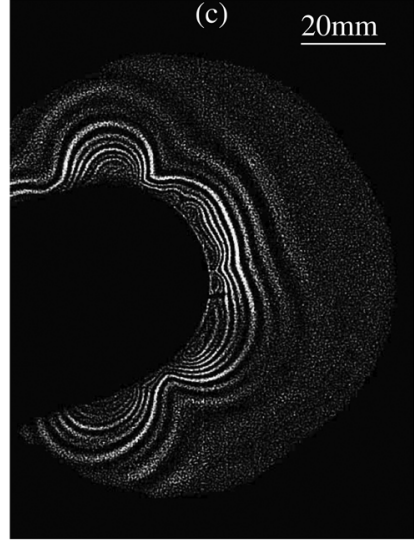

$\mathrm{t}=4 \mathrm{~s}$

Stable state with the increase of third-body flow

Fig. 3 Evolution of isochromatics during the creation of third-body particles for a radial expansion of $20 \mu \mathrm{m}$ and a rotation speed $\omega=0.2 \mathrm{~m} / \mathrm{s}$
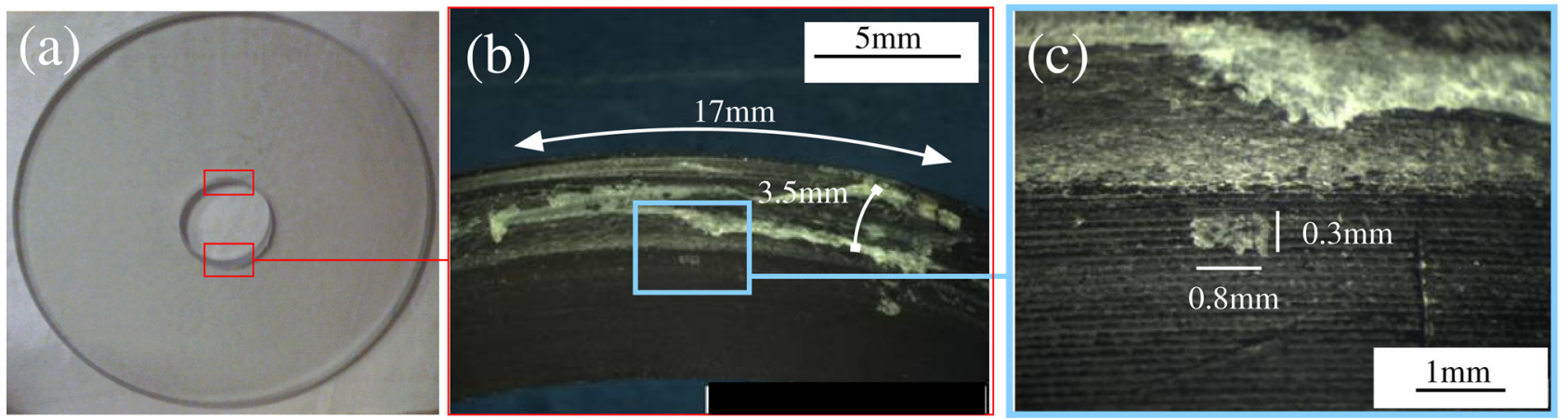

Fig. 4 Zoom on third-body particles on the inner diameter disk with a binocular microscope 
particles is directly highlighted thanks to the local increase of the number of fringes (cf. Figs. 3(b) and 3(c)) on the isochromatics. The rotation of the cylinder leads to the creation and the migration of third-body particles within the contact. At the location point of third-body particles, $\tau_{\max }$ raises at values about 10 times higher than its initial value (up to approximately $7 \mathrm{MPa}$ ).

2.4 Third-Body Particles. After a few seconds, the contact instability disappears (no more squeal) and the rotation of the cylinder is stopped. Using the video camera, it is possible to visualize the history of the contact interface. With the rotation of the cylinder, third-body particles are created. When the amount of thirdbody particles is large enough, contact instability vanishes. Consequently, the inner diameter of the cylinder is observed with a stereo microscope (cf. Fig. 4) in order to identify the shape of third-body particles.

Third-body particles present different shapes and different sizes. Two main kinds of particles are here distinguished: large particles and small particles. The size of large particles is not negligible compared to the size of the contact: several millimeters width, several tens or so millimeters length, and several tens or so micrometers thickness. The size of "small particles" is equal to about 100 micrometers, with a thickness of a few micrometers. As these last ones are also observed during experiments when the instability remains, large particles can be seen as the agglomeration of small particles and are the cause of instability disappearance.

\section{Numerical Approach}

3.1 Global Formulation. Several observations have been performed thanks to the experimental setup: contact instabilities, evolution of local stresses, and creation of third-body particles. Such phenomena are deeply related and occur at different scales. In order to use the same numerical approach to model the whole disk and the third-body particles, the choice of a combined FEM-DEM formulation has been done [13]: finite elements to model the polycarbonate disk and discrete elements to represent third-body particles.

The combined FEM-DEM approach relies on the nonsmooth contact dynamics (NSCD) method developed by Moreau and Jean $[18,19]$. Based on a robust mathematical framework, the approach is able to represent, with the same formalism, the dynamical behavior of large collections of rigid particles as well as deformable particles [13]. Only the headlines of the approach are presented here, and the reader could refer to dedicated papers for more details $[18,19]$.

When the evolution of a multicontact system is smooth, the equation of dynamics can be written as

(a)

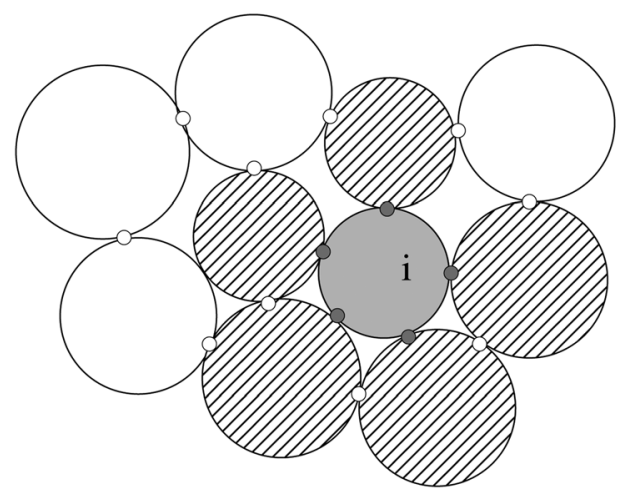

$$
\mathbb{M} \ddot{\mathbf{q}}+\mathbb{C} \dot{\mathbf{q}}+\mathbb{K} \mathbf{q}=\mathbf{F}^{\mathrm{ext}}(t, \mathbf{q}, \dot{\mathbf{q}})+\mathbf{R}
$$

where $\mathbb{M}$ represents the mass matrix, $\mathbb{C}$ the damping matrix, $\mathbb{K}$ the stiffness matrix, $\mathbf{F}^{\text {ext }}(t, \mathbf{q}, \dot{\mathbf{q}})$ the external forces, and $\mathbf{R}$ the contact forces. Vector $\mathbf{q}$ represents the vector of generalized degrees of freedom, while $\dot{\mathbf{q}}$ and $\ddot{\mathbf{q}}$ denote the generalized velocity and acceleration vectors, respectively. The Eq. (1) is written for deformable as well as rigid bodies. In this last case, both $\mathbb{C}$ and $\mathbb{K}$ vanish.

The NSCD approach uses an implicit time-discretized formulation of the equation of motion in Eq. (1) written for a collection of particles. Since the number of contacts is higher than the number of particles (dense particle assemblies), the interaction between particles is not considered at the scale of each individual particle but at each contact scale. Consequently, one prefers to transform the Eq. (1) in an equation where local variables are used.

Two linear mappings, $\mathbb{H}$ and $\mathbb{H}^{*}$, are defined to related variables expressed in the frame of particles (global frame) and variables expressed in the frame of contacts (local frame). In a contact between two rigid particles (cf. Fig. 5(b)), information is exchanged between the center of mass of each particle (black points) and the contact point (gray point) while in a contact between a rigid particle and a deformable structure, information is exchanged between the center of mass of the particle and the nodes of the closer segment (black points) and the contact point (gray point). Thus, in both cases, the local contact force vector $\mathbf{r}$ and the local relative velocity vector $\mathbf{u}$ are related to the global body contact force vector $\mathbf{R}$ and the body velocity vector $\dot{\mathbf{q}}$ by

$$
\left\{\begin{array}{l}
\mathbf{R}=\mathbb{H} \mathbf{r} \\
\mathbf{u}=\mathbb{H}^{*} \dot{\mathbf{q}}
\end{array}\right.
$$

Both mappings depend on local information, such as the local frame defined at each contact point and the network connectivity of each contact. The combination to the Eq. (1) and the equations of system (2) allows us to write, at each time step, the system in Eq. (3) contains equations of motion,

$$
\left\{\begin{array}{l}
\mathbb{W} \mathbf{r}-\mathbf{u}=\mathbf{b} \\
\operatorname{law}_{\alpha}\left[\mathbf{u}_{\alpha}, \mathbf{r}_{\alpha}\right]=\text { true }, \alpha=1, n_{c}
\end{array}\right.
$$

where matrix $\mathbb{W}$ is the Delassus operator, containing local information (such as local frames and contact points), allowing us to use it at the particles scale. Vectors $\mathbf{u}$ and $\mathbf{r}$ are, respectively, the vectors containing the relative velocity and the mean contact impulse for all the contact points of all particles. b represents the free relative velocity calculated by taking into account the (b)

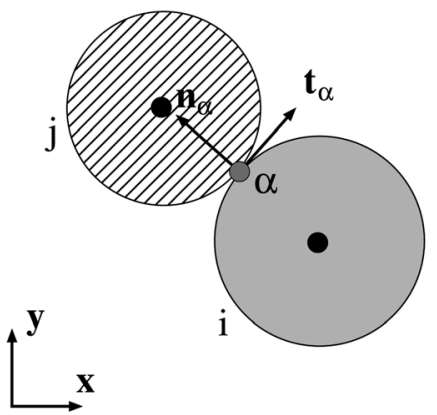

(c)

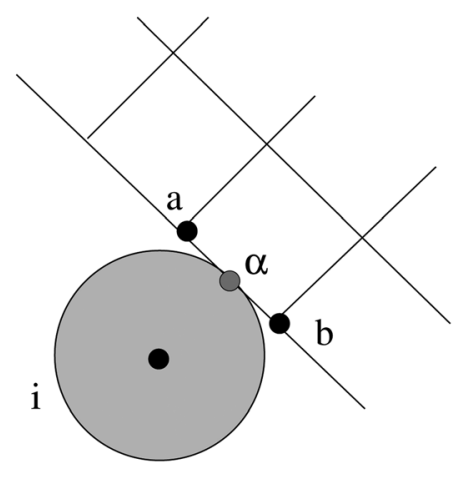

Fig. 5 Definition of the list of first neighbors of particle $i$ (dashed particles) (a) and of the local frame $\left(t_{\alpha}, n_{\alpha}\right)$ for a contact between two particles $(b)$ and between one particle and a deformable structure $(c)$ 
external forces only. The first equation of system in Eq. (3) represents, for a given time, the connection between the elements of the mechanical system submitted to some external forces represented by $\mathbf{b}$, while the second one explains how each couple of particle (rigid or now) are related. The $\theta$ method is used for time discretization and a classical nonlinear Gauss-Seidel algorithm to solve the system in Eq. (3) [19]. The approach benefits from a parallel version to ensure reasonable central processing unit times [20].

3.2 Interaction Laws. To close the system in Eq. (3), one should define the relation between $\mathbf{u}$ and $\mathbf{r}$ given by the interaction law. This could represent a unilateral contact, an elastic contact, a bilateral relation, or a more sophisticated relation. In our case, two kinds of interaction laws should be defined.

The first one concerns the contact between the polycarbonate disk and the steel cylinder. A classical unilateral contact law coupled with a Coulomb friction model is used, involving only the friction coefficient $\mu$ as a parameter.

The second one concerns the contact between different discrete elements, between discrete elements and the disk, and between discrete elements and the cylinder. No information can be directly obtained to fix such a law. Nevertheless, it is possible to interpret the phenomena within the contact according to postmortem observation: Macroparticles are a collection of microparticles that also can be represented as a collection of elementary rigid particles. Using a kind of cohesive interaction law, it is possible to reproduce such phenomena as well as to allow for a deformation of microparticles. Consequently, as proposed in previous works $[3,11,13]$, a cohesive unilateral contact law is used, involving the cohesion force $\gamma$ as a parameter. This cohesive law can be combined with the Coulomb friction to introduce tangential effects.

The system in Eq. (4) summarizes the previous laws,

$$
\left\{\begin{array}{lll}
\left(r_{n}+\gamma\right) \geq 0, & u_{n} \geq 0, & \left(r_{n}+\gamma\right) \cdot u_{n}=0 \\
\text { If } u_{t}=0, & \text { then } & \left|r_{t}\right| \leq \mu r_{n} \\
& \text { else } & r_{t}=-\operatorname{sign}\left(u_{t}\right) \mu r_{n}
\end{array}\right.
$$

where the indices $n$ and $t$ refer to the normal and tangential parts of the considered vector.

3.3 Structural Damping. The role of the damping is crucial to determine the dynamical response of a given system. For this reason, it is necessary to account for the structural damping rather than the artificial numerical one. A Rayleigh model [21] is chosen to describe the structural damping. Such damping is related to the damping matrix $\mathbb{C}$, which can be represented as

$$
\mathbb{C}=\alpha_{R} \mathbb{M}+\beta_{R} \mathbb{K}
$$

where $\alpha_{R}$ and $\beta_{R}$ are the Rayleigh's coefficients.

To obtain structural damping coherent values, dynamical tests have been performed on a polycarbonate disk: The disk is excited by an instrumented hammer and its dynamical response is measured with an accelerometer. Such an experiment, by fitting the modal damping values obtained with the half-power method [17], allows us to recover the corresponding values of the Rayleigh's coefficients: $\alpha=40 s^{-1}$ and $\beta=4.5 e-7 s$.

3.4 Numerical Model. To reproduce the experimental setup, a two-dimensional numerical model is used. Due to contrast between the Young's modulus of the steel and the polycarbonate, the inner cylinder is represented as a rigid disk. The polycarbonate disk is represented by a mesh constituted of 15,400 elements Q4 (four linear integration points), where the length of a cell close to the contact is equal to $0.58 \mathrm{~mm}$. Material properties (Young's modulus, Poisson ratio, density, and radii) are the same as in the experiment (cf. Table 1).

Two numerical third-body macroparticles are used in the combined FEM-DEM model and located in the areas A and B presented in Fig. 6, corresponding to the areas observed experimentally in Fig. 4. As mentioned above, small particles do not affect contact instabilities. Consequently, only macroparticles are taking into account in the numerical model. They are both composed of rigid disks of diameter equal $20 \mu \mathrm{m}$. The initial thickness of macroparticles (in the radial direction) is equal to $50 \mu \mathrm{m}$, i.e., two particles high approximately. The macroparticles $\mathrm{A}$ and $\mathrm{B}$ are composed of 203 and 628 rigid particles, respectively. The rigid disks contained in each macroparticle are related by the cohesive unilateral contact law defined by the system in Eq. (4). As such a cohesive law is reversible, macroparticles could be split and recombined during the simulation process.

Like for the experimental tests, simulations are performed in two steps. First, the internal rigid cylinder has a radius expansion of $0.15 \mathrm{~mm}$. Then, the expansion is stopped and the rotation of the cylinder starts with an angular velocity $\omega$ equal to $6.25 \mathrm{rad} / \mathrm{s}$ equivalent to a linear velocity of $0.1 \mathrm{~m} / \mathrm{s}$ at the contact interface.

To close this subsection, one should determine the value of contact law parameters, i.e., the value of $\mu$ and $\gamma$ in the different contact configurations.

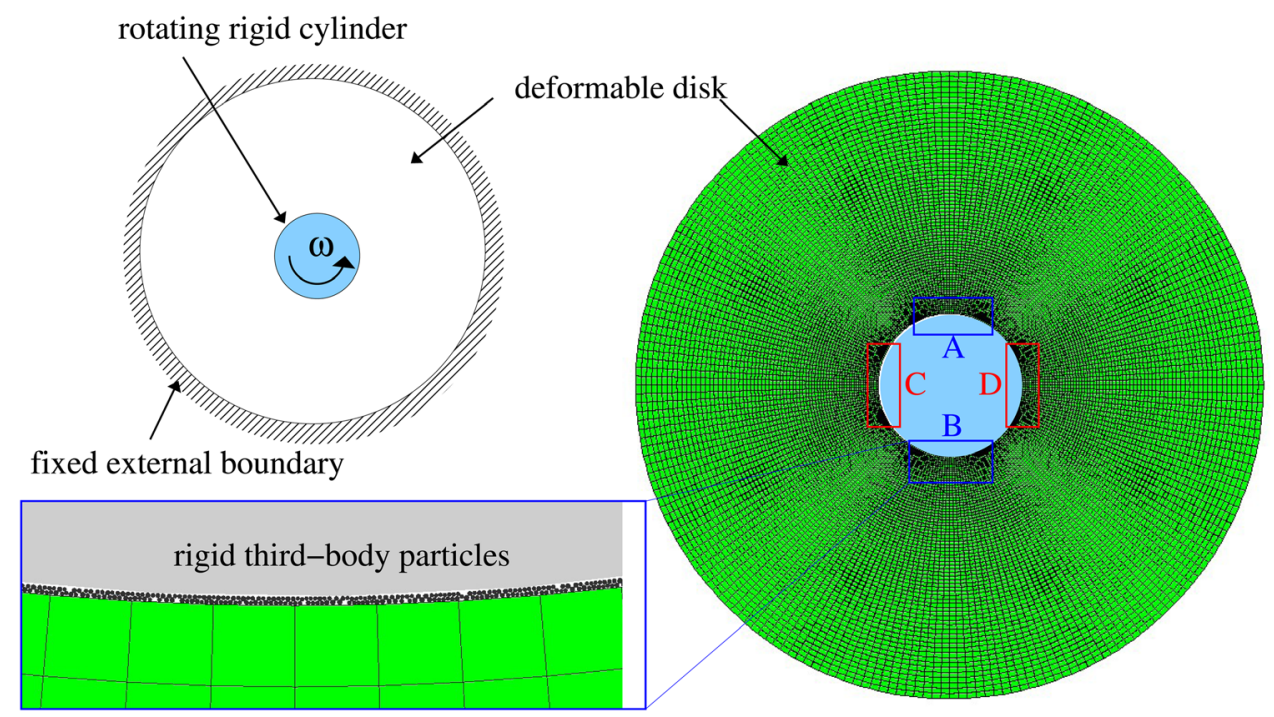

Fig. 6 Numerical model used for standalone FEM and the combined FEM-DEM simulations 


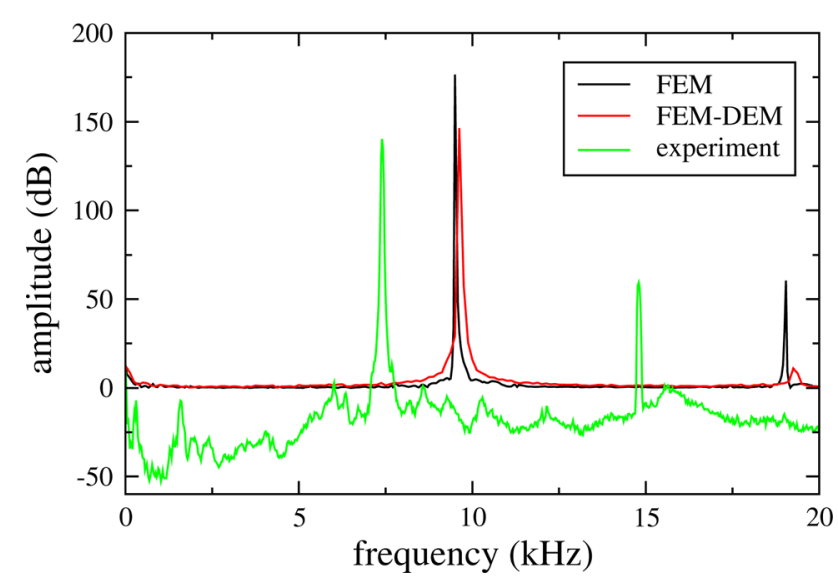

Fig. 7 Frequency calculated on the standalone FEM model and the combined FEM-DEM model during the frictional process compared to the experimental result

In the literature $[22,23]$, the friction value for a contact steel/ polycarbonate ranges from 0.3 to 0.7 . Nevertheless, a previous numerical study [24] shows that, for angular velocity equal to $6.25 \mathrm{rad} / \mathrm{s}$, the value of $\mu$ does not affect the instability regimes. Consequently, a value of 0.3 is taken for $\mu$ and is combined with a dry contact law (i.e., $\gamma=0$ ). The same parameters are chosen for the contact between discrete elements and the steel cylinder.

Concerning the contact between discrete elements and between discrete elements and the deformable disk, a value of 0.3 is taken for $\mu$ and is combined with four different cohesion values: $0,0.1$, 0.2 , and $1.0 \mathrm{~N}$. Such variation of the cohesion could characterize implicitly some variation in the particle properties (surface energy for example). The maximal value of $1 \mathrm{~N}$ is chosen to obtain an internal resistance of macroparticle equivalent to the traction resistance value of the polycarbonate (close to $60 \mathrm{MPa}$ ) [23].

\section{Result Discussion and Comparisons}

4.1 Without Third-Body. During the simulation, when no third-body particles are considered, the trajectory of a node located in the area $\mathrm{D}$ is measured during the frictional process and the spectrum of the signal is computed (cf. Fig. 7). The same harmonic behavior of the system vibrations is recovered both numerically and experimentally. The standalone FEM modeling shows a frequency at about $9.5 \mathrm{kHz}$.

This value, higher than the experimental one, is due to the ideal numerical boundary conditions (fixed support) and thus to higher rigidity of the model with respect to the experimental setup. A 3D numerical modal analysis of the whole setup has been performed to verify that the numerical and experimental unstable modes coincide [17]. In complement of such a value, the deviatoric stress fields are observed and compared directly with experimental observations (cf. Fig. 8).

There is a good correlation between the experimental and numerical results. The shape of the excited mode is similar in simulations and experiments: the contour levels of the deviatoric stress fields have the same repartition and the same maximal value.

4.2 With Third-Body. In complement of Sec. 4.1, comparisons have also been realized between numerical and experimental results in presence of third-body particles. As for the standalone FEM model, the trajectory of a node located in the area D is measured during the frictional process and the frequency spectrum is calculated (cf. Fig. 7). The combined FEM-DEM modeling shows a frequency close to $9.7 \mathrm{kHz}$. Then, the deviatoric stress fields are observed and compared directly with experimental observation (cf. Fig. 9).

Once again, there is a good correlation between the experimental and numerical results. Even in presence of third-body particles, the contour levels of the deviatoric stress fields of the disk have the same maximal value. In Fig. 9, the circled numbers correspond to the order of fringe of the photoelasticimetry analysis. By multiplying such number by $0.35 \mathrm{MPa}$ (according to the properties of the polycarbonate disc), the deviatoric stress is obtained and could so be compared to the isostresses lines presented on the FEM image. A quantitative agreement is found between experimental results and numerical results at the scale of the disc.

Finally, in complement to the comparison between experimental and numerical results, the influence of third-body behavior on its global evolution is investigated. To analyze third-body rheology, the contact interface is divided in 220 angular sectors, denoted $\mathcal{S}_{k}$, each sector corresponding to a cell of the mesh. Then, at each time step, particles are associated to a given sector according to their current position. Finally, the average of quantities, such as the velocity magnitude or equivalent deviatoric

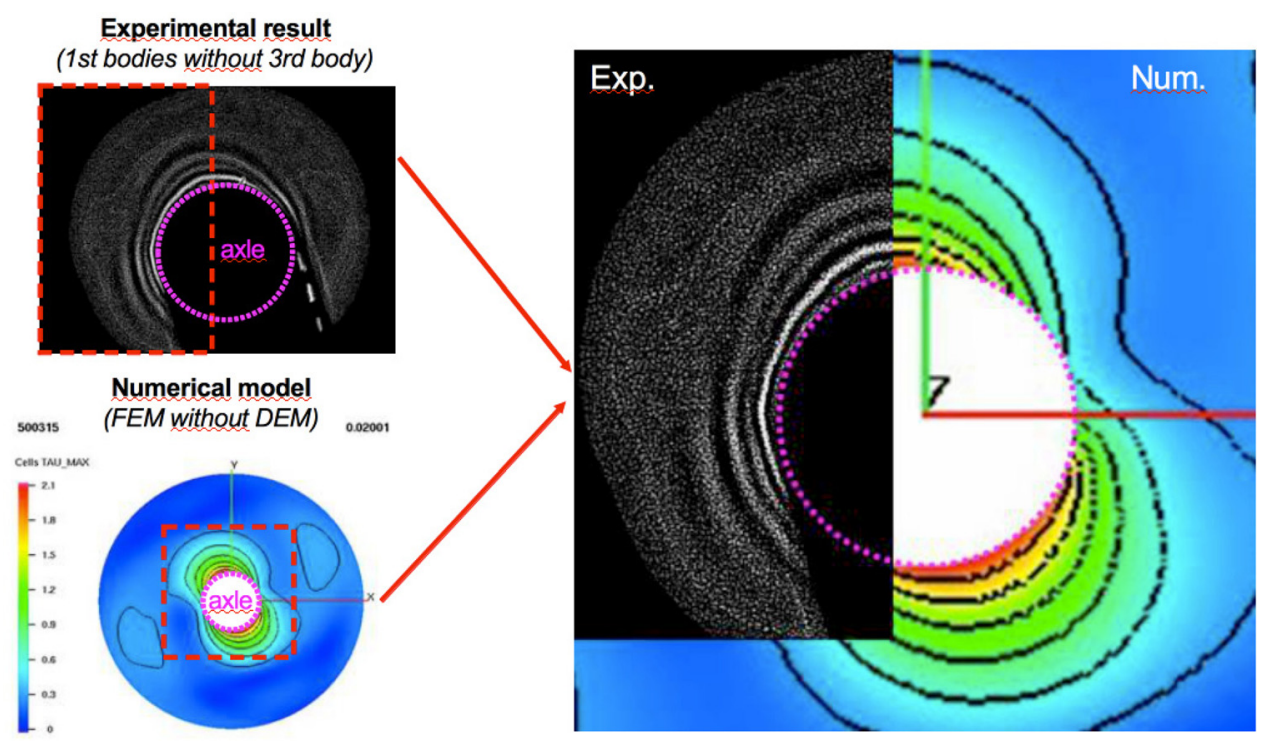

Fig. 8 Comparison of the deviatoric stress values between the FEM-DEM model and experimental results without third-body (cf. Fig. 3(a)). 


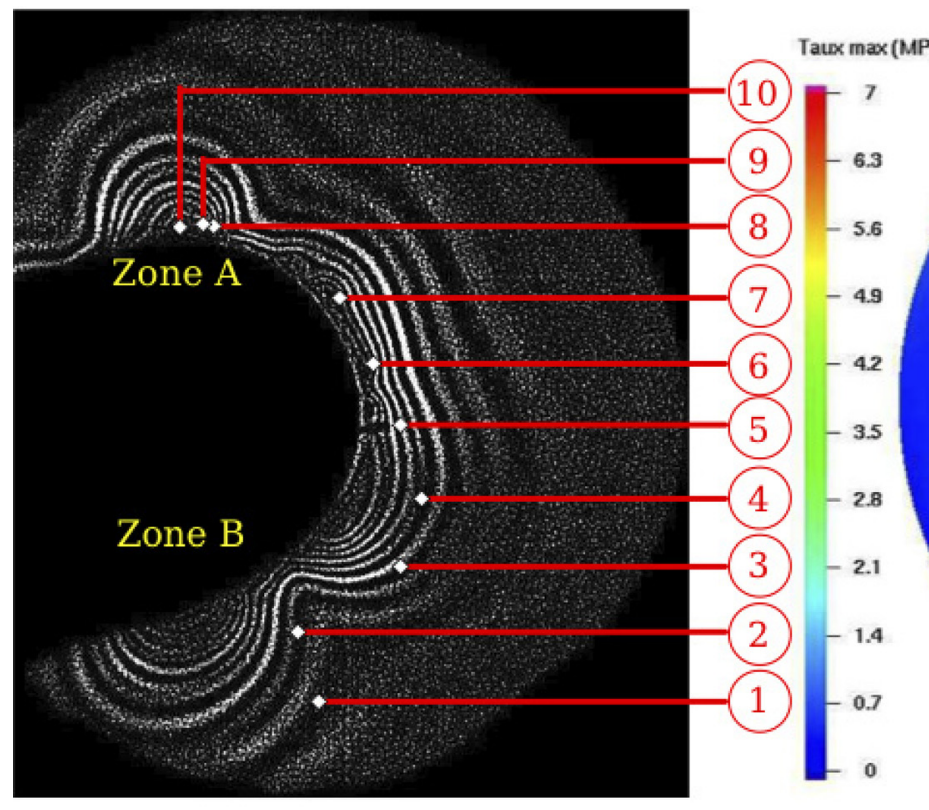

a)

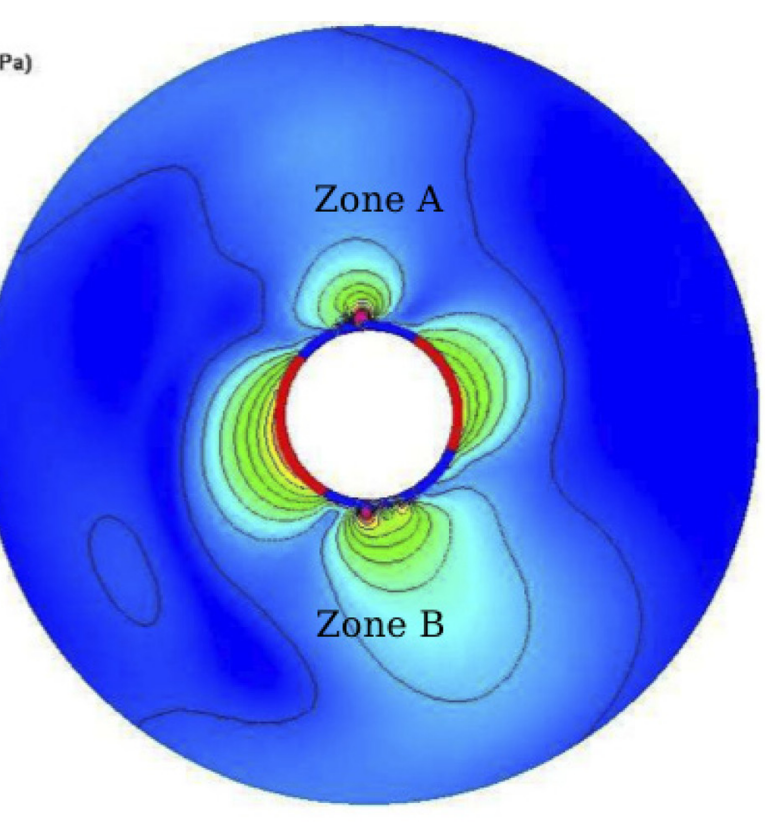

b)

Fig. 9 Comparison of the deviatoric stress field between the FEM-DEM model and experimental results with third-body. The numbers from 1 to 10 correspond to the number of fringes identified in the experiment.

stress [25], is computed. The velocity magnitude of a cell is The equivalent deviatoric stress is defined as defined as

$$
V\left(\mathcal{S}_{k}\right)=\frac{1}{n_{k}} \sum_{i \in \mathcal{S}_{k}} \dot{\mathbf{q}}_{i}
$$

where $n_{k}$ denotes the number of particles in the sector $\mathcal{S}_{k}$.

$$
\tau\left(\mathcal{S}_{k}\right)=\sum_{i \in \mathcal{S}_{k}} \tau_{i}
$$

where $\tau_{i}$ is the deviatoric part of the mean stress tensor $\sigma_{i}$ of particle $i$ computed as follows:

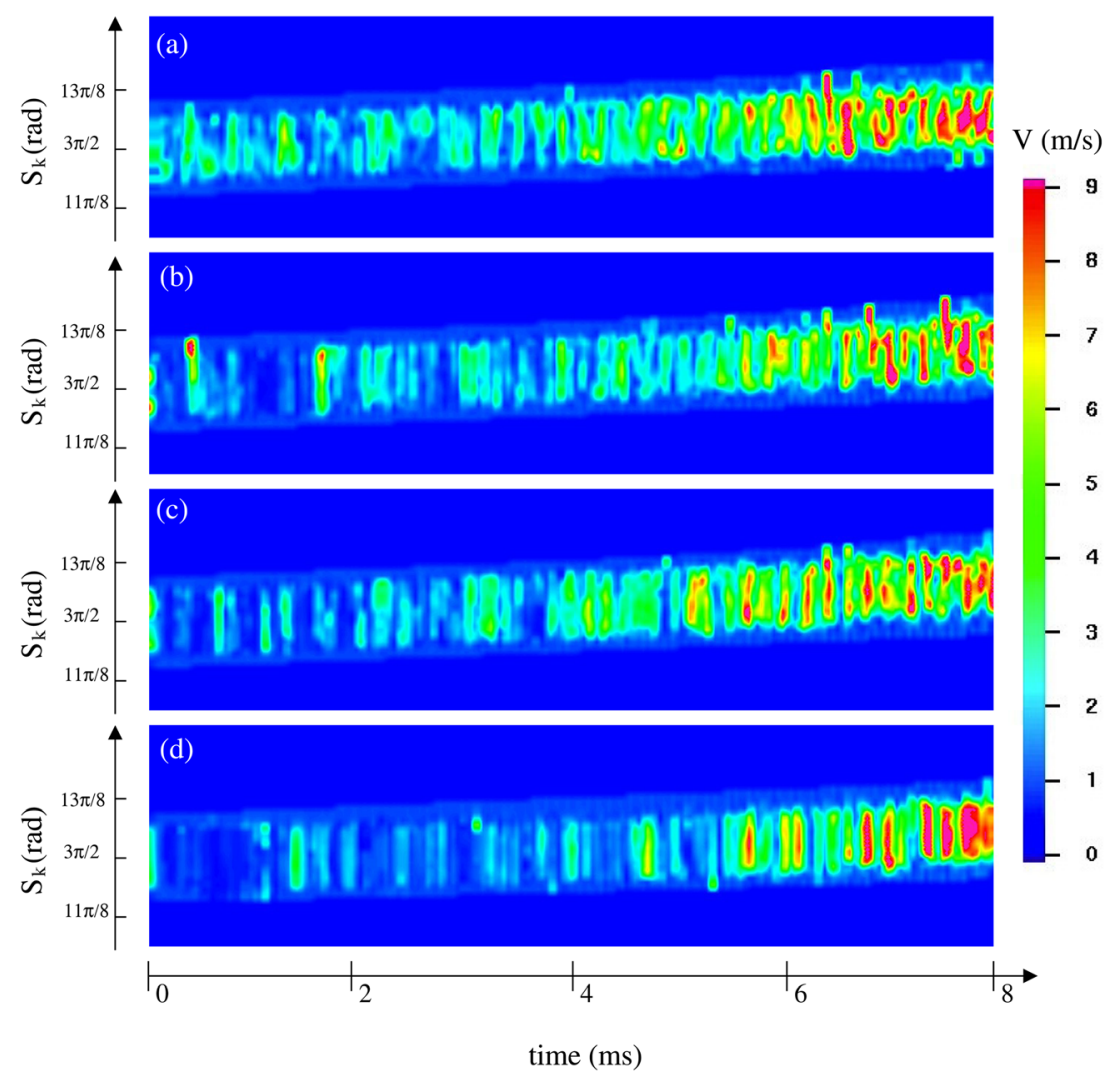

Fig. 10 Visualization of the equivalent velocity field within a macroparticle during the process for different cohesion values: (a) $0,(b) 0.1,(c) 0.2$, and (d) 1.0 


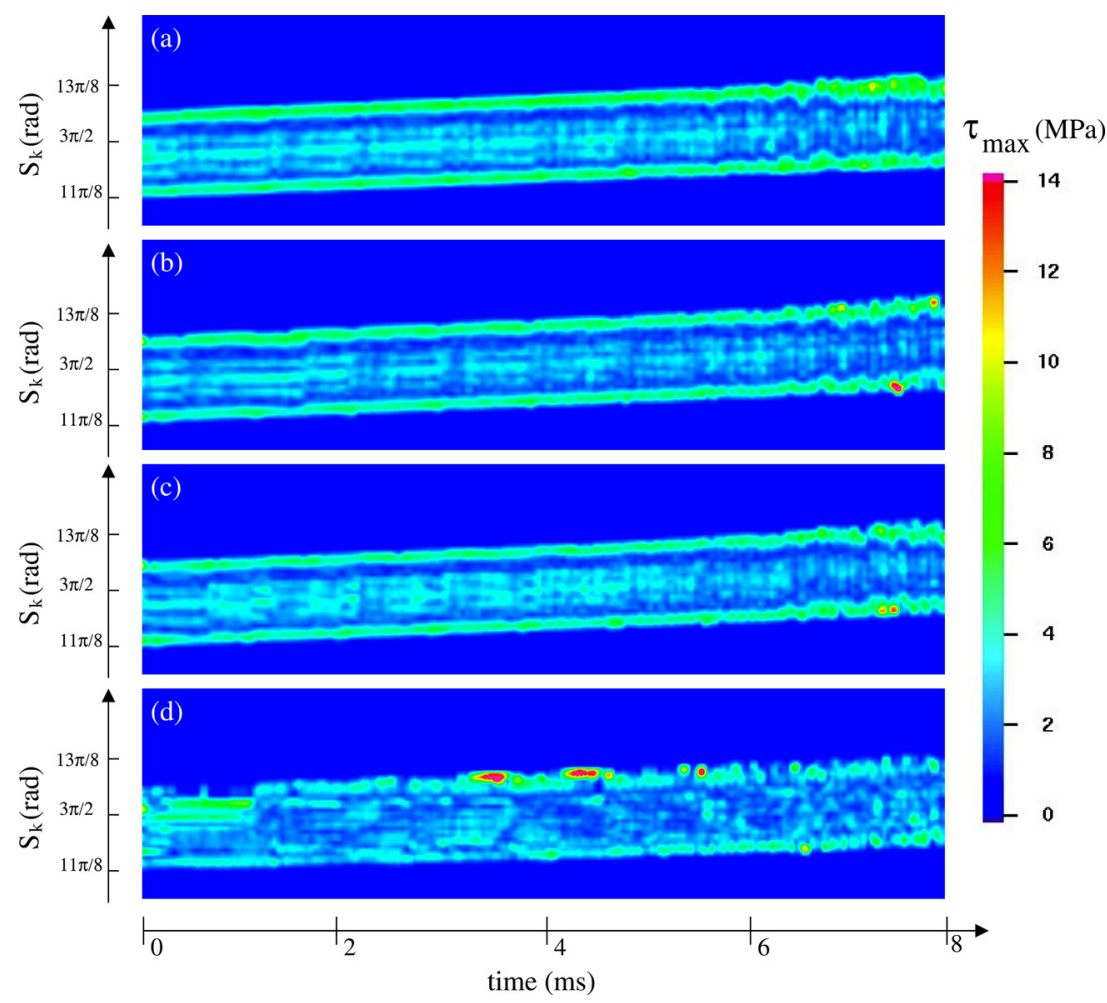

Fig. 11 Visualization of the equivalent deviatoric stress within a macroparticle during the process for different cohesion values: $(a) 0,(b) 0.1,(c) 0.2$, and $(d) 1.0$

$$
\sigma_{i}=\frac{1}{A_{i}} \sum_{\alpha \in \mathcal{L}_{i}} \mathbf{r}_{\alpha} \otimes \mathbf{l}_{\alpha}
$$

where $A_{i}$ is the area of particle $i$ and $\mathcal{L}_{i}$ the contact list of particle $i$ (dashed particles of Fig. 5(a)), while $\mathbf{l}_{\alpha}$ is the vector connecting the center of mass of particle $i$ and the contact point associated to the contact force $\mathbf{r}_{\alpha}$.

Figure 10 proposes the visualization of the equivalent velocity field within a macroparticle during its motion induced by the rotation of the inner cylinder for the different cohesion values.

It can be noted that the value of the cohesion does not affect the migration of the particle. In each case, the macroparticle occupies initially 26 angular sectors and 29 at the end of the simulation. Indeed, the velocity within the particle could be six times larger than the rotation speed. This observation underlines the fact that internal motion occurs within a macroparticle, even if its global shape does not evolve. Such motion could be related to a selfreorganization or to a "vibration" induced by the wave propagation in the polycarbonate disk. The harmonic vibrations due to the modal instability cause large oscillations of the local sliding velocity at the contact interface. In such a way, the dynamic response of the system affects the local behavior (kinematics) of the third-body particles. This is confirmed by the period of the jumps of the macroparticles, which coincide with the period of the harmonic system vibrations. Its variation is closer to an increasing oscillatory function rather than to a linear one. This last point underlines the strong impact of the dynamics of the first bodies on the rheology of the third-body. Moreover, the comparison between the three plots, obtained for different values of the cohesiveness, shows that its effect on this period is not relevant with respect to the effect of the system vibrations, which appears to be dominant.

To complete local kinematic observations, Fig. 11 presents the visualization of the equivalent deviatoric stress within a macroparticle.

As highlighted in the figures, only the higher cohesion value affects the stress repartition within the particle. For small cohesion values, the deviatoric stress reaches its maximal value at the limits of the particle (the front and the end) during the whole frictional process. For the higher cohesion value, this repartition starts to be chaotic, especially for the front of the particle. In term of stress intensity, the striking point is the fact that the mean computed value within the particle is equal to the one computed in the continuous model, which corresponds to the experimental one. Thus, there is a really good agreement between the discrete, the continuous models, and the experiment. One can observe in Fig. 11 deviatoric stress values two times larger than the mean deviatoric stress value. This points out a limit of a model constituted of only rigid particles that can generate higher constraint values and suggests the introduction of local fragmentation process in the simulation to reduce such an artifact.

\section{Conclusion}

In the present work, a combined FEM-DEM approach is proposed to account for both a good description of the bodies in contact and the contact interface. Simulation results are not only phenomenological but also qualitatively validated thanks to the comparison with an experimental setup. The setup, dedicated to a tribological model, has been developed to reproduce and measure both instability states and third-body flows using a frictional contact between a holed polycarbonate disc and an expanded inner cylinder. From an experimental point of view, a competition between instability states and third-body flows in the contact has been highlighted. From a numerical point of view, local investigation can be performed on third-body particles to check the influence of internal parameters, such as the cohesion value.

\section{Acknowledgment}

This work is a part of the project DiNEET (Dialogue Numérique Entre Echelles Tribologiques - ANR-JCJC-080020) funded by the ANR (Agence National de la Recherche) program. 
Simulations are performed with the LMGC90 open-source platform. ${ }^{1}$

\section{Nomenclature}

$\mathbf{b}=$ free local relative velocity

$\mathbb{C}=$ damping matrix

$d t=$ time step

$g=$ gap between particles

$i=$ particle index

$k=$ angular sector index

$\mathbb{K}=$ stiffness matrix

$\mathbb{M}=$ mass matrix

$n_{c}=$ contact number

$P=$ contact pressure

$\mathbf{q}=$ configuration parameter

$\dot{\mathbf{q}}=$ global velocity

$\ddot{\mathbf{q}}=$ global acceleration

$\mathbf{r}=$ local contact force

$\mathbf{R}=$ global contact force

$\mathcal{S}=$ angular sector

$\mathbf{u}=$ local relative velocity

$\mathbb{W}=$ Delassus operator of the whole system

$\alpha=$ contact index

$\alpha_{R}, \beta_{R}=$ Rayleigh coefficients

$\gamma=$ local cohesion force

$\mu=$ local friction coefficient

$\omega=$ angular relative velocity of the disk

$\sigma=$ stress tensor

$\tau_{\max }=$ deviatoric stress

\section{References}

[1] Berthier, Y., 1990, "Experimental Evidence for Friction and Wear Modelling," Wear, 139, pp. 77-92.

[2] Saulot, A., Descartes, S., Desmyter, D., Levy, D., and Berthier, Y., 2006, “A Tribological Characterization of the Damage Mechanism of Low Rail Corrugation on Sharp Curved Track," Wear, 260(9-10), pp. 984-995.

[3] Richard, D., Iordanoff, I., Renouf, M., and Berthier, Y., 2008, "Thermal Study of the Dry Sliding Contact With Third Body Presence," ASME J. Tribol., 130(3), p. 031404

[4] Raje, N., Sadeghi, F., Rateick, R. G., and Hoeprich, M. R., 2007, "Evaluation of Stresses Around Inclusions in Hertzian Contacts Using the Discrete Element Method," ASME J. Tribol., 129(2), pp. 283-291.

[5] Godet, M., 1990, "Third-Bodies in Tribology," Wear, 136(1), pp. 29-54.
[6] Adams, G. G., 1995, "Self-Excited Oscillations of Two Elastic Half-Spaces Sliding With a Constant Coefficient of Friction," ASME J. Appl. Mech., 62(4), pp. 867-872.

[7] Ranjith, K., and Rice, J. R., 2001, "Slip Dynamics at an Interface Between Dissimilar Materials," J. Mech. Phys. Solids, 49, pp. 341-361.

[8] Di Bartolomeo, M., Massi, F., Baillet, L., Culla, A., Fregolent, A., and Berthier, Y., 2012, "Wave and Rupture Propagation at Frictional Bimaterial Sliding Interfaces: From Local to Global Dynamics, From Stick-Slip to Continuous Sliding," Tribol. Int., 52, pp. 117-131.

[9] Peillex, G., Baillet, L., and Berthier, Y., 2008, "Homogenization in Non-linear Dynamics Due to Frictional Contact," Int. J. Solid Struct., 45(9), pp. 2451-2469.

[10] Elrod, H., and Brewe, D., 1991, "Numerical Experiments With Flows of Elongated Granules," Tribol. Ser., 21, pp. 219-226.

[11] Iordanoff, I., Seve, B., and Berthier, Y., 2002, "Solid Third Body Analysis Using a Discrete Approach: Influence of Adhesion and Particle Size on Macroscopic Properties,” ASME J. Tribol., 124(3), pp. 530-538.

[12] Temizer, I., and Wriggers, P., 2008, "A Multiscale Contact Homogenization Technique for the Modeling of Third Bodies in the Contact Interface," Comput. Methods Appl. Mech. Eng., 198(3-4), pp. 377-396.

[13] Cao, H.-P., Renouf, M., Dubois, F., and Berthier, Y., 2011, "Coupling Continuous and Discontinuous Descriptions to Model First Body Deformation in Third Body Flows,” ASME J. Tribol., 133(4), p. 041601.

[14] Leonard, B. D., Patil, P., Slack, T. S., Sadeghi, F., Shinde, S., and Mittelbach, M., 2011, "Fretting Wear Modeling of Coated and Uncoated Surfaces Using the Combined Finite-Discrete Element Method," ASME J. Tribol., 133(2), p. 021601.

[15] Tonazzi, D., Massi, F., Culla, A., Baillet, L., Fregolent, A., and Berthier, Y. "Instability Scenarios Between Elastic Media Under Frictional Contact," Mech. Syst. Signal Process. (in press).

[16] Renouf, M., Massi, F., Fillot, N., and Saulot, A., 2011, "Numerical Tribology of a Dry Contact," Tribol. Int., 44(7-8), pp. 834-844.

[17] Renouf, M., Massi, F., Saulot, A., and Fillot, N., 2012, "Dialogue Numérique Entre Echelles Tribologiques," ANR JC Tech. Rep.

[18] Moreau, J. J., 1994, "Some Numerical Methods in Multibody Dynamics: Application to Granular Materials," Eur. J. Mech. A Solids, 13(4-suppl.), pp. 93-114.

[19] Jean, M., 1999, "The Non-smooth Contact Dynamics Method," Comput. Methods Appl. Mech. Eng., 177(3-4), pp. 235-257.

[20] Renouf, M., Dubois, F., and Alart, P., 2004, "A Parallel Version of the Non Smooth Contact Dynamics Algorithm Applied to the Simulation of Granular Media," J. Comput. Appl. Math., 168(1-2), pp. 375-382.

[21] Mohammad, D., and Khan, N., 1995, "On the Role of Rayleigh Damping," J. Sound Vib., 185(2), pp. 207-218.

[22] Lee, J., Xu, G., and Liang, H., 2001, "Experimental and Numerical Analysis of Friction and Wear Behavior of Polycarbonate," Wear, 251(1-12), pp. $1541-1556$.

[23] Mergler, Y., Kampen, R. V., Nauta, W., Schaake, R., Raas, B., Griensven, J. V., and Meesters, C., 2005, "Influence of Yield Strength and Toughness on Friction and Wear of Polycarbonate," Wear, 258(5-6), pp. 915-923.

[24] Linck, V., Baillet, L., and Berthier, Y., 2003, "Modeling the Consequences of Local Kinematics of the First Body on Friction and on Third Body Sources in Wear," Wear, 255(1-6), pp. 299-308.

[25] Bagi, K., 1996, "Stress and Strain in Granular Assemblies," Mech. Mater. 22(3), pp. 165-177. 